\title{
Design of Tunable Flat-top Bandpass Filter Based on Two Long-period Fiber Gratings and Core Mode Blocker
}

\author{
Jinho Bae* \\ Department of Ocean System Engineering, Jeju National University, Jeju 690-756, Korea \\ Junkye Bae \\ Fiber Access 3 Team, LG-Ericsson CO., Ltd. LG R\&D Complex, Anyang 431-749, Korea \\ Sang Bae Lee \\ Nanophotonics Research Center, KIST, Seoul 130-650, Korea
}

(Received March 23, 2011 : revised May 17, 2011 : accepted May 18, 2011)

\begin{abstract}
We propose a tunable flat-top bandpass filter to pass light in a customized wavelength band by using long-period fiber gratings (LPFG) structure. The LPFG structure is composed of a core mode blocker in between two LPFGs. The bandpass spectrum of the proposed structure is obtained in overlapped wavelength band of two LPFGs operating on the same modes. To analyze the properties, we introduce a mathematical matrix model for the structure. We theoretically demonstrate flexibility of the flat-top bandpass filter with various bandwidths.
\end{abstract}

Keywords: Flat-top bandpass, Long-period fiber gratings, Core mode blocker, Matrix model

OCIS codes : (350.2770) Gratings; (350.2460) Filters, interference; (230.2285) Fiber devices and optical amplifiers; (070.1170) Analog optical signal processing

\section{INTRODUCTION}

In long-period fiber gratings (LPFG), a fundamental core mode and the multiple cladding modes are coupled, which all propagate in the same (forward) direction. Due to unique features of low insertion loss, low back-reflection and excellent polarization insensitivity, LPFGs have attracted great interest in the optical communications and sensor applications [1, 2]. Some researchers have studied the manufacture of the core mode blocker [3, 4], band pass filter using the core mode blocker [5-7], and tunable bandpass filter using coil heater [8, 9].

The core mode blocker is a device to block off the propagated light in the core, and pass the propagated light in the cladding. Some researchers have developed bandpass filters using LPFGs, and some of them have studied the topics based on a core mode blocker as shown in Fig. 1. In Fig. 1, the $\mathrm{LPFG}_{1}$ is used to interact between core and cladding at resonant wavelength, and light existing in the core is extinguished by the core mode blocker. The light in the resonant wavelength is propagated through the cladding to the second LPFG. The resonant light existing in the cladding by the $\mathrm{LPFG}_{2}$ is transferred back into the core. The central points of the research are to study the physical phenomenon [3, 4] and the manufacture of the bandpass filter [5-7, 9]. However the design of the flat-top bandpass filter [11] with specific customized wavelength band using an accurate analysis model was not proposed yet.

In the paper, we present the matrix model to analyze the

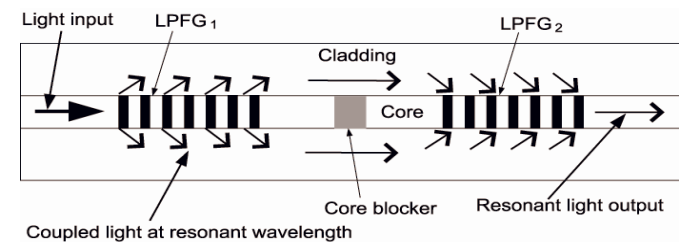

FIG. 1. The LPFG structure with core mode blocker.

\footnotetext{
*Corresponding author: baejh@jejunu.ac.kr

Color versions of one or more of the figures in this paper are available online.
} 
LPFG structures with the core mode blocker. The proposed matrix model is a useful mathematical tool to describe the properties of the structure. We analyze the physical properties of the LPFG structure with a core mode blocker by using the proposed model. By using specially coupled feature of only the same modes in overlapped wavelength region, we demonstrate the design of the flat-top bandpass filter with the customized bandwidth.

\section{THE PROPOSED MATHEMATICAL MATRIX MODEL}

The structure in Fig. 1 is modeled as the FIR (Finite Impulse Response) filter block diagram as shown in Fig. 2(a). Figure 2(b) is the signal flow graph to analyze both $\mathrm{LPFG}_{1}$ and $\mathrm{LPFG}_{2}$, which is called the multiport lattice filter model [10-12]. Here, $D_{1}$ and $D_{2}$ are the delay of the diagonal matrix, $C B$ is matrix for core mode blocker, $E_{c o}$ and $E_{d l}^{(i)}$ are the E-fields (electric fields) of the fundamental core mode and the $i$ th cladding mode, respectively. And $\beta_{c o}$ and $\beta_{c l}^{(i)}$ are the propagation constant of the fundamental core mode and the $i$ th cladding mode for the LPFGs, $L_{1}$ and $L_{2}$ are the length of $\mathrm{LPFG}_{1}$ and $\mathrm{LPFG}_{2}$, and $d_{l}, d_{2}$, and $\mathrm{d}_{b}$ are the length for $D_{1}, D_{2}$, and $C B$.

In Fig. 2(a), the E-fields coming into and out from the structure can be written to be

$$
\left[\begin{array}{c}
E_{c o}(\text { out }) \\
E_{c l}^{(1)}(\text { out }) \\
\vdots \\
E_{c l}^{(N)}(\text { out })
\end{array}\right]=Q\left[\begin{array}{c}
E_{c o}(\text { in }) \\
E_{c l}^{(1)}(\text { in }) \\
\vdots \\
E_{c l}^{(N)}(\text { in })
\end{array}\right], \quad Q \equiv M_{2} D_{2} C B D_{1} M_{1}
$$

where $D_{k}=\operatorname{diag}\left(e^{-j \beta_{c o} d_{k}}, e^{-j \beta_{c l}^{(1)} d_{k}}, \cdots e^{-j \beta_{c l}^{(N)} d_{k}}\right),(k=1,2)$ are diagonal matrices and $C B=\operatorname{diag}\left(0, e^{-j \beta_{c l}^{(1)} d_{b}}, \cdots e^{-j \beta_{c l}^{(l)} d_{b}}\right)$ is a diagonal matrix with zero determinant.

By assuming the $\mathrm{LPFG}_{\mathrm{k}},(k=1,2)$ are uniform long period fiber gratings, we can easily get the matrix form of the $(\mathrm{N}+1) \times(\mathrm{N}+1)$ complex matrix $M_{k},(k=1,2)$ for the $\mathrm{LPFG}_{\mathrm{k}}$ with codirectional interactions since the $\mathrm{LPFG}_{\mathrm{k}}$ has the multiport lattice filter structure in [10] as follows:

$$
M_{k} \equiv D_{G} G^{(N)} G^{(N-1)} \cdots G^{(1)},
$$

where $G^{(p)}$ is $(\mathrm{N}+1) \times(\mathrm{N}+1)$ identity matrix except for the four entries adopted from $F^{(\mathrm{p})}$ in Fig. 2(b). The relation between $G^{(\mathrm{p})}$ and $F^{(\mathrm{p})}$ are as follows:

$$
\begin{aligned}
& G_{1,1}^{(p)}=F_{1,1}^{(p)}, \quad G_{1, p+1}^{(p)}=F_{1,2}^{(p)}, \quad G_{p+1,1}^{(p)}=F_{2,1}^{(p)}=-\left(F_{1,2}^{(p)}\right)^{*}, \\
& G_{p+1, p+1}^{(p)}=F_{2,2}^{(p)}=\left(F_{1,1}^{(p)}\right)^{*} .
\end{aligned}
$$

(Here, $\mathrm{B}_{\mathrm{k}, 1}$ denotes the $(k, l)$ element of the matrix B.) The matrix $D_{\mathrm{G}}$ represents the phase shift of the $\mathrm{LPFG}_{2}$

$$
\begin{aligned}
& D_{G}= \operatorname{diag}\left(e^{-j \beta_{c o} L_{k}}, e^{-j \beta_{c l}^{(1)} L_{i}}, \cdots e^{-j \beta_{c l}^{(N)} L_{k}}\right) \text { and } \\
& F^{(p)} \equiv\left[\begin{array}{cc}
\left(\cos \left(S_{p} L_{k}\right)-j \frac{\delta_{p}}{S_{p}} \sin \left(S_{p} L_{k}\right)\right) e^{j \delta_{p} L_{k}} & \left.-j \frac{K_{p}}{S_{p}} \sin \left(S_{p} L_{k}\right)\right) e^{j \delta_{p} L_{k}} \\
\left.-j \frac{K_{p}}{S_{p}} \sin \left(S_{p} L_{k}\right)\right) e^{-j \delta_{p} L_{k}} & \left(\cos \left(S_{p} L_{k}\right)+j \frac{\delta_{p}}{S_{p}} \sin \left(S_{p} L_{k}\right)\right) e^{-j \delta_{p} L_{k}}
\end{array}\right],
\end{aligned}
$$

and $S_{p} \equiv \sqrt{\left(K_{p}\right)^{2}+\left(\delta_{p}\right)^{2}}, L_{k}$ is the length of the LPFG ${ }_{\mathrm{k}}, \delta_{p}$ is the detuning factor for the $p$ th cladding mode, and $\kappa_{p}$ is the coupling coefficient between the fundamental core mode and the $p$ th cladding mode [10-13].

Note that $M_{k}$ in (2) can be regarded as a multiport lattice section. Because of the decoupling property of modes, the matrices $G^{(\mathrm{p})}$ in $M_{k}$ commute with each other so that the

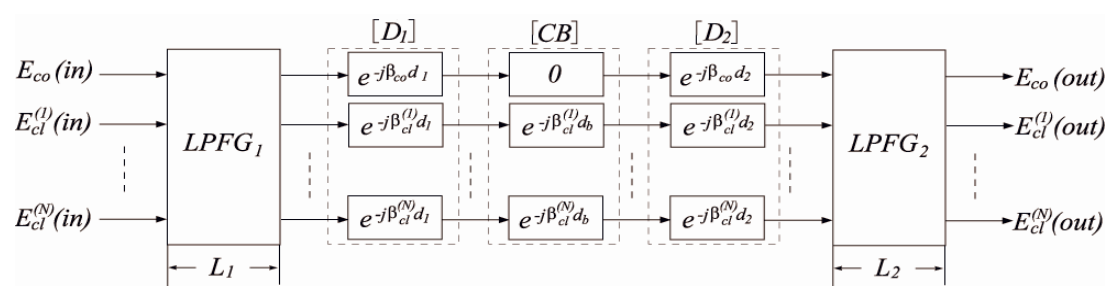

(a)

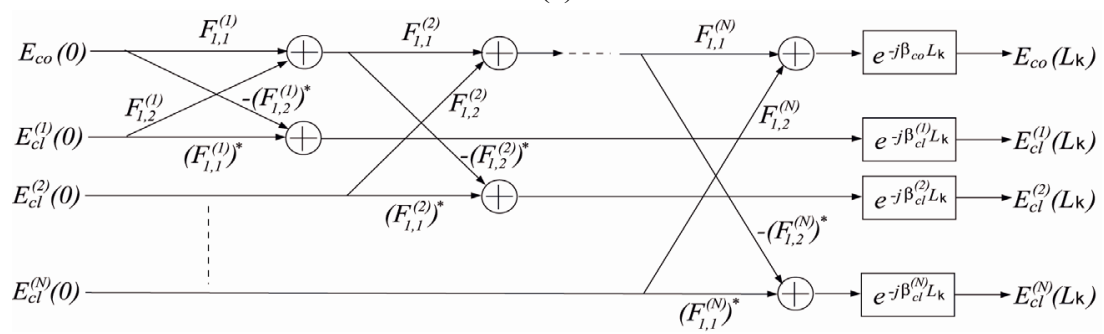

(b)

FIG. 2. (a) An equivalent block diagram for Fig. 1. (b) Multiport lattice filter model. 
$2 \times 2$ subsections may be interchanged freely. If $\mathrm{LPFG}_{1}$ and $\mathrm{LPFG}_{2}$ are extended as piecewise uniform LPFGs, we can also easily get the $M_{k}$ using the multiport lattice filter model [10]. Now, the overall transmission coefficient $t \equiv E_{c o}($ out $) / E_{c o}($ in $)$ with $E_{c l}^{(i)}($ in $)=0,(1 \leq i \leq N)$ is easily seen to be $t=Q_{1, l}$, which is the $(1,1)$-element of $Q$.

As shown in Fig. 2(a), after the coupled light on the $\mathrm{LPFG}_{1}$ is absorbed at $C B$, and then in the $\mathrm{LPFG}_{2}$, the propagated light in the cladding is coupled with the same modes from cladding to core, due to the orthogonality of modes as shown in Fig. 2(b) [10]. Because the overall transmission is calculated as the complex multiplying of two bandpass filters, we cannot get the spectrum in the core if two filters have entirely different bandpass regions.

\section{EXAMPLES}

We analytically calculated the transmission spectrum curves for the concatenated LPFGs with a core mode blocker in the following examples by computing the transmission coefficient $t=Q_{l, l}$ in (1). The parameters of the fiber used in these examples are as follows:

$n_{c o}=1.449, n_{c l}=1.444, n_{a i}=1, r_{c o}=4.5 \mu \mathrm{m}, r_{c l}=62.5 \mu \mathrm{m}$ where $n_{c l}$ is the refractive index of the cladding, $n_{\text {air }}$ is the refractive index of air, $r_{c o}$ is the radius of the core, and $r_{c l}$ is the radius of the cladding.

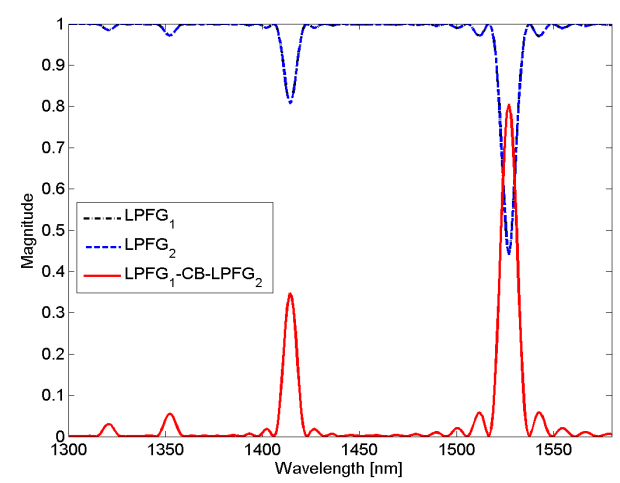

(a)

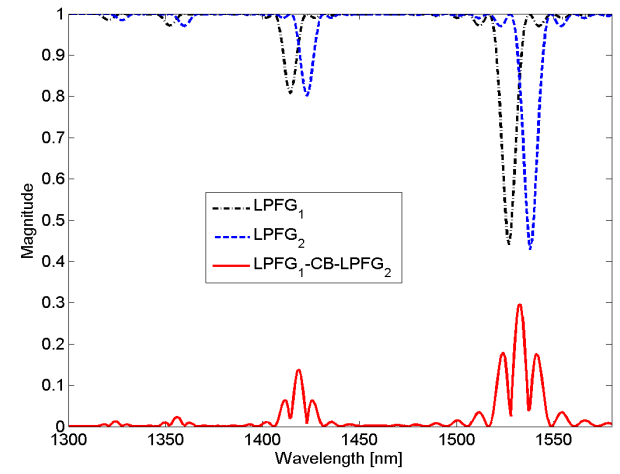

(c)

\subsection{Example 1 for Uniform LPFG with Both Core Mode Blocker and Delay}

Figure 3 shows the transmission for the modes $L P_{0 i},(i$ $=1, \cdots, 4)$ in the wavelength range between $1300 \mathrm{~nm}$ and $1580 \mathrm{~nm}$, where $\mathrm{LPFG}_{1}$ and $\mathrm{LPFG}_{2}$ have uniform gratings. Their lengths are $L_{1}=100 \Lambda_{1}$ and $L_{2}=100 \Lambda_{2}$, respectively, the grating period for $\mathrm{LPFG}_{1}$ is $\Lambda_{1}=441.24 \mu \mathrm{m}, \Lambda_{2}$ for $\mathrm{LPFG}_{2}$ is varied (441.24 $\mu \mathrm{m}$ for Fig. 3(a), $443.44 \mu \mathrm{m}$ for Fig. 3(b), $445.65 \mu m$ for Fig. 3(c), and $501.28 \mu m$ for Fig. 3(d)) and their induced index changes are $\Delta n_{1}=n_{2}=0.00011$, respectively. The length of the $\mathrm{D}_{1}$ and the $\mathrm{D}_{2}$ are $d_{1}=400$ $\Lambda_{1}$ and $d_{2}=400 \Lambda_{1}$, respectively. The length of the $C B$ is assumed to be $d_{b}=0$.

To analyze the properties of the concatenated LPFGs with the core mode blocker, we have calculated the transmission spectra along the change of the $\Lambda_{2}$ as shown in Fig 3. Figures 3(a)-3(c) show the transmission along the overlapped region of the spectrum band of the same modes. We can only get the bandpass spectra by coupling the same modes in the overlapped region. Figure 3(d) shows the transmission for the coupling with the different modes $\left(L P_{04}\right.$ of $L P F G_{1}$ and $L P_{03}$ for $\left.L P F G_{2}\right)$. From the result, we know that the bandpass filter cannot be obtained from coupling the different modes.

\subsection{Example 2 for Flat-top Bandpass Filters}

We can design a flat-top bandpass filter with the customized bandwidth using the property of coupling with the same modes and the overlapped band. We have synthesized

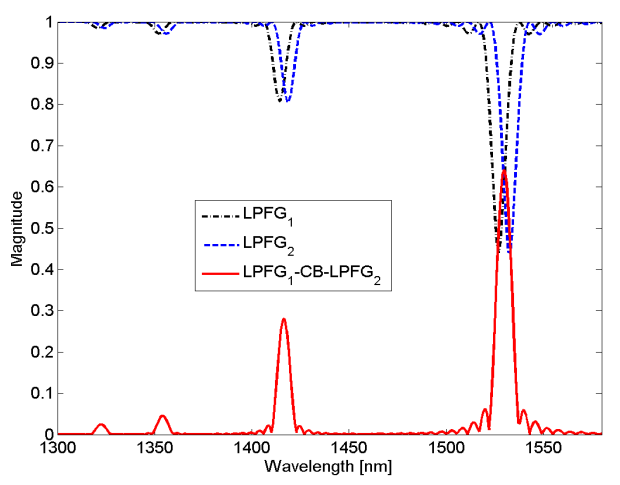

(b)

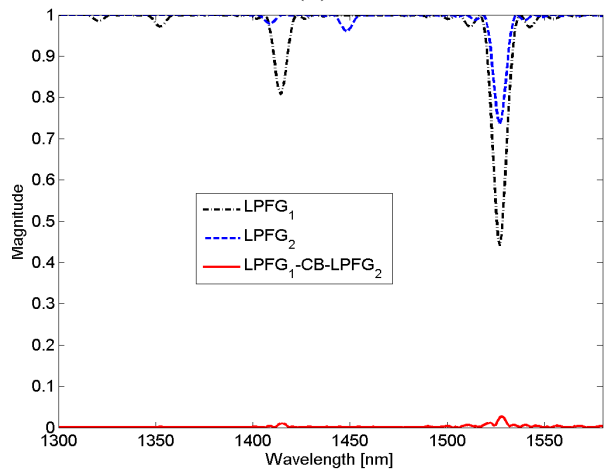

(d)

FIG. 3. $\Lambda_{1}=441.24 \mu m$ is fixed and $\Lambda_{2}$ is changed ((a) $\Lambda_{2}=441.24 \mu m$, (b) $\Lambda_{2}=443.44 \mu m$ (c) $\Lambda_{2}=445.65 \mu m$ and (d) $\Lambda_{2}=501.28 \mu m$ ). 


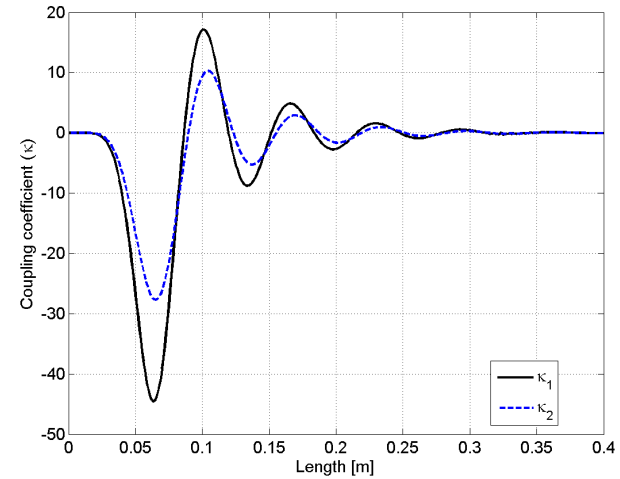

(a)

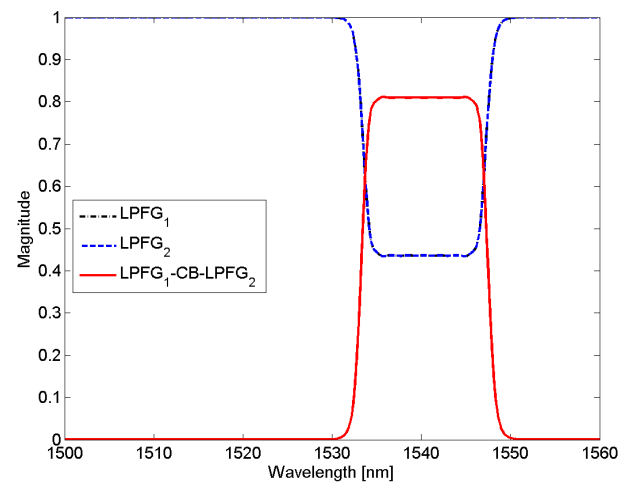

(c)

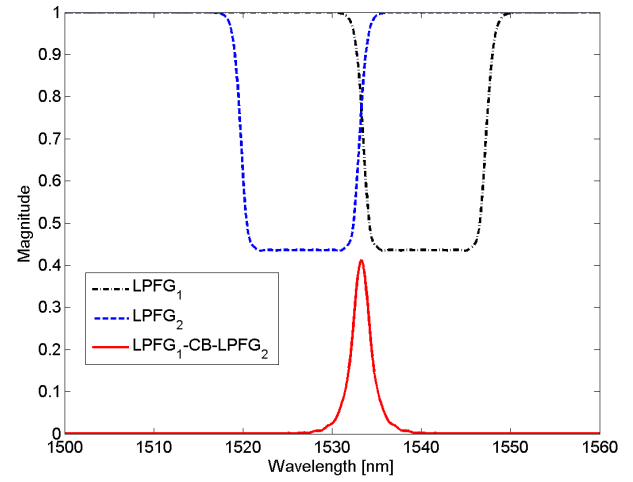

(e)

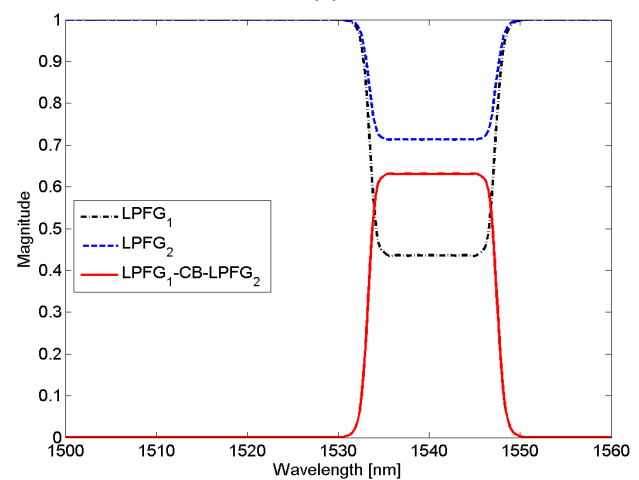

(g)

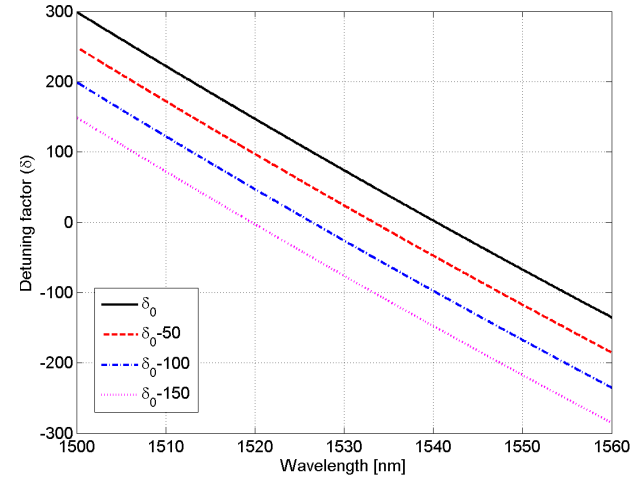

(b)

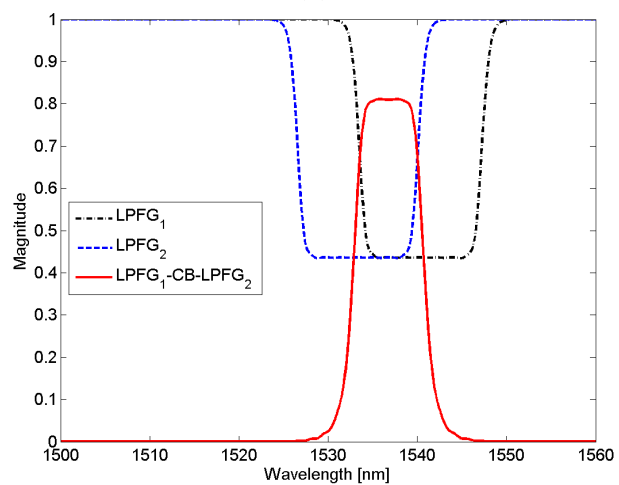

(d)

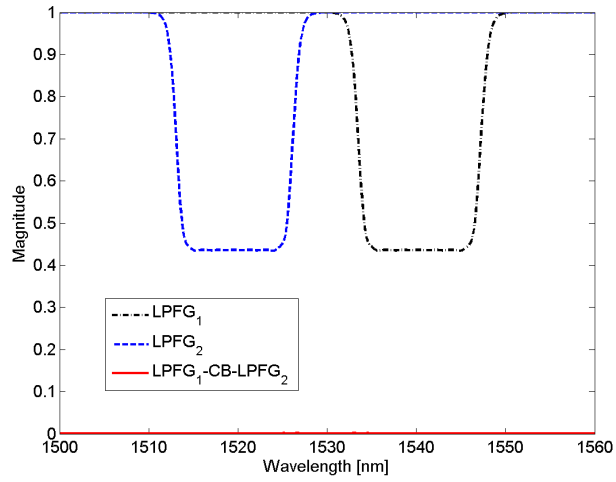

(f)

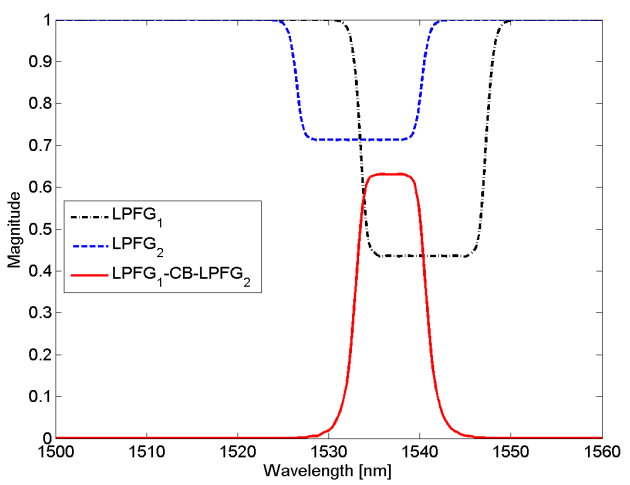

(h)

FIG. 4. (a) Coupling coefficients. (b) Detuning factors. (c) The $\kappa_{1}$ and the $\delta_{0}$ are utilized for the $\mathrm{LPFG}_{1}$ and the $\mathrm{LPFG}_{2}$. (d) $\mathrm{LPFG}_{1}\left(\kappa_{1}\right.$ and $\delta_{0}$ is used.) and $\mathrm{LPFG}_{2}\left(\kappa_{1}\right.$ and $\delta_{0}-50$ is used.). (e) $\mathrm{LPFG}_{1}\left(\kappa_{1}\right.$ and $\delta_{0}$ is used.) and $\mathrm{LPFG}_{2}\left(\kappa_{1}\right.$ and $\delta_{0}-100$ is used.). (f) $\mathrm{LPFG}_{1}\left(\kappa_{1}\right.$ and $\delta_{0}$ is used.) and $\mathrm{LPFG}_{2}\left(\kappa_{1}\right.$ and $\delta_{0}-150$ is used.). (g) $\mathrm{LPFG}_{1}\left(\kappa_{1}\right.$ and $\delta_{0}$ is used.) and $\mathrm{LPFG}_{2}\left(\kappa_{2}\right.$ and $\delta_{0}$ is used.). (h) $\mathrm{LPFG}_{1}\left(\kappa_{1}\right.$ and $\delta_{0}$ is used.) and $\mathrm{LPFG}_{2}\left(\kappa_{2}\right.$ and $\delta_{0}-50$ is used.). 
the tunable flat-top filter by considering a single cladding mode as shown in Fig. 4. We have utilized the coupling coefficients $\kappa$ and the detuning factors $\delta$, respectively, as shown in the Figs. 4(a) and 4(b). The coupling coefficients in Fig. 4(a) can be found by the Gel'fand-Levitan-Marchenko coupled equations $[14,15]$.

The $\kappa_{1}$ in Fig. 4(a) is used for the $\mathrm{LPFG}_{1}$ and $\mathrm{LPFG}_{2}$ of Figs. 4(c)-4(f). In Fig. 4(b), the $\delta_{0}$ is used for the $\mathrm{LPFG}_{1}$ and $\mathrm{LPFG}_{2}$ is used the $\delta_{0}$ for Fig. $4(\mathrm{c})$, the $\delta_{0}-50$ for Fig. 4(d), the $\delta_{0}-100$ for Fig. $4(\mathrm{e})$, and the $\delta_{0}-150$ for Fig. 4(f). We have assumed the delay is $d_{1}=d_{2}=0$ and the core mode blocker is $C B=\operatorname{diag}(0,1,1 \cdots, 1)$, ideally. The $3 \mathrm{~dB}$ bandwidth of the $\mathrm{LPFG}_{1}$ and $\mathrm{LPFG}_{2}$ is $13.9 \mathrm{~nm}$. In the case, we can control the bandwidth of the flat-top bandpass filter in the wavelength range less than $13.9 \mathrm{~nm}$ $\pm \varepsilon$ (where $\varepsilon$ is a small variation by calculating the equation (1).). The $3 \mathrm{~dB}$ bandwidth of the obtained flat-top filter in Figs. 4(c) and 4(d) are $14.2 \mathrm{~nm}(\varepsilon=0.3 \mathrm{~nm})$ and $8 \mathrm{~nm}$, respectively.

From the results, we can observe that the bandwidth of the bandpass is changed along the overlapped band region of the flat-top band-rejection of both $\mathrm{LPFG}_{1}$ and $\mathrm{LPFG}_{2}$. If we can make the ideal band-rejection filter, we can synthesize a sharp bandpass filter with the customized bandwidth as well as the bandpass filters with a tunable bandwidth. Especially, if the bandwidth of $\mathrm{LPFG}_{1}$ and $\mathrm{LPFG}_{2}$ aren't overlapped as shown in Fig. 4(f), we cannot synthesize the desired filter.

We also demonstrated the transmission along the overlapped region, when the coupling coefficients are different as shown in Figs. 4(g) and 4(h). The coupling coefficients $\kappa_{1}$ for the $\mathrm{LPFG}_{1}$ and $\kappa_{2}$ the $\mathrm{LPFG}_{2}$ are utilized as shown in Fig. 4(a). The detuning factors $\delta$ for Figs. 4(g) and 4(h) are the same ones as for Figs. 4(c) and 4(d). We have also used the delay and the core mode blocker as above examples.

From the Figs 4(g) and 4(h), we have obtained similar results to Figs. 4(c) and 4(d) although the $\mathrm{LPFG}_{1}$ and $\mathrm{LPFG}_{2}$ have different magnitude levels. The $3 \mathrm{~dB}$ bandwidth of the $\mathrm{LPFG}_{2}$ is $14.1 \mathrm{~nm}$. The $3 \mathrm{~dB}$ bandwidth of the obtained flat-top filter in Figs. 4(c) and 4(d) are 14.2 $n m(\varepsilon=0.1 \mathrm{~nm})$ and $8 \mathrm{~nm}$, respectively. The results show that the magnitude level of the flat-top bandpass filter is dependent on the magnitudes of the $\mathrm{LPFG}_{1}$ and $\mathrm{LPFG}_{2}$.

\section{CONCLUSION}

The analytical model for the LPFG with the core mode blocker is proposed. We have also analyzed and described the physical phenomenon of the structure by using the proposed model. We have proposed a design method of the flat-top bandpass filter with customized bandwidth. We have also demonstrated, through computer simulations, the bandwidth of the flat-top filters controlled by tuning the overlapped band region.

\section{ACKNOWLEDGMENT}

This work was supported by the research grant from the Chuongbong Academic Research Fund of Jeju National University in 2010.

\section{REFERENCES}

1. A. Othonos and K. Kalli, Fiber Bragg Gratings - Fundamentals and Applications in Telecommunications and Sensing (Artech House, Boston, USA, 1999).

2. S. Kim, G. H. Kim, K. Hwang, S. D. Lim, K. Lee, S. H. Kim, S. B. Lee, and J. Jeong, "Investigation of an arcinduced long period fiber grating inscribed in a photonic crystal fiber with two large air holes," J. Opt. Soc. Korea 13, 428-433 (2009).

3. Y. G. Han, S. H. Kim, S. B. Lee, U. C. Paek, and Y. Chung, "Development of core mode blocker with $\mathrm{H}_{2}$-loaded Ge-B codoped fibres," Electron. Lett. 39, 1107-1108 (2003).

4. S. D. Lim, J. G. Kim, K. Lee, S. B. Lee, and B. Y. Kim, "Fabrication of a highly efficient core-mode blocker using a femtosecond laser ablation technique," Opt. Express 17, 18449-18454 (2009).

5. S. Choi, T. J. Eom, J. W. Yu, B. H. Lee, and K. Oh, "Novel all-fiber bandpass filter based on hollow optical fiber," IEEE Photon. Technol. Lett. 14, 1701-1703 (2002).

6. S. Choi, T. J. Eom, J. W. Yu, and B. H. Lee, "Broad-band tunable all-fiber bandpass filter based on hollow optical fiber and long-period grating pair," IEEE Photon. Technol. Lett. 17, 115-117 (2005).

7. H. Sakata, Y. Takata, and S. Suzuki, "Single-channel bandpass filter based on vernier-aligned long-period fiber gratings," IEEE Photon. Technol. Lett. 19, 1661-1663 (2007).

8. D. S. Starodubov, V. Grubsky, and J. Feinberg, "All-fiber bandpass filter with adjustable transmission using claddingmode coupling," IEEE Photon. Technol. Lett. 10, 1590-1592 (1998).

9. Y. Han, S. H. Kim, and S. B. Lee, "Flexibly tunable multichannel filter and bandpass filter based on long-period fiber gratings," Opt. Express 12, 1902-1907 (2004).

10. J. Bae, J. Chun, and S. B. Lee, "Synthesis of long-period fiber gratings with the inverted erbium gain spectrum using the multiport lattice filter model," J. Ligthwave Technol. 22, 1976-1986 (2004).

11. H. Kim, J. Bae, and J. Chun, "Synthesis of flat-top bandpass filters using two-band rejection long-period fiber gratings," IEEE Photon. Technol. Lett. 19, 1466-1468 (2007).

12. H. Kim, J. Bae, J. W. Lee, J. Chun, and S. B. Lee, "Analysis of concatenated longperiod fiber gratings having phase-shifted and cascaded effects," Jpn. J. Appl. Phys. Part 1 42, 5098-5101 (2003).

13. M. Yi, Y. Kim, D. Yee, and J. Ahn, "Terahertz frequency spreading filter via one-dimensional dielectric multilayer structures,” J. Opt. Soc. Korea 13, 398-402 (2009).

14. G. Song and S. Shin, "Design of corrugated waveguide filters by the Gel'fand-Levitan-Marchenko inverse-scattering method," J. Opt. Soc. Am. A 2, 1905-1915 (1985).

15. E. Peral, J. Capmany, and J. Marti, "Iterative solution to the Gel'fand-Levitan-Marchenko coupled equations and application to synthesis of fiber gratings," J. Quantum Electron. 32, 2078-2084 (1996). 